\title{
Variational Approach to transport in quantum dots
}

\author{
Nicola Lanatà ${ }^{1}$ \\ ${ }^{1}$ University of Gothenburg, SE-412 96 Gothenburg, Sweden
}

(Dated: November 18, 2018)

\begin{abstract}
We have derived a variational principle that defines the nonequilibrium steady state transport across a correlated impurity (mimicking e.g. a quantum dot) coupled to biased leads. This variational principle has been specialized to a Gutzwiller's variational space, and applied to the study of the simple single-orbital Anderson impurity model at half-filling, finding a good qualitative accord with the observed behavior in quantum dots for the expected regime of values of the bias. Beyond the purely theoretical interest in the formal definition of a variational principle in a nonequilibrium problem, the particular methods proposed have the important advantage to be simple and flexible enough to deal with more complicated systems and variational spaces.
\end{abstract}

PACS numbers: 74.20.Mn, 71.27.+a, 71.30.+h, 71.10.Hf

\section{INTRODUCTION}

Nanocontacts of quantum dots, single molecules or atoms, and nanowires are ideal candidates to realize electronic devices where a source-drain current across the contact can be magnetically controlled. Indeed, because of the low dimensionality of the contact region, electronic correlations grow in strength and may stabilize a local magnetism that influences electron tunneling. The Kondo-like zero-bias anomalies first observed in quantum dots 1 are just the simplest manifestation of such a local magnetism, but one can foresee even more spectacular phenomena, like giant magnetoconductance ${ }^{2}$

From the theory side, this is a complicated problem first of all because electronic correlation is the main actor and is difficult to treat, and secondly because the inelastic tunneling spectrum requires full out-of-equilibrium calculations. Many complementary techniques have been used to characterize the nanocontact at equilibrium. For instance ab initio Local Density Approximation (LDA) calculations can provide the electronic structure and predict whether magnetism could indeed be stabilized, ${ }^{2-4}$ at least at the mean field level. Inclusion of quantum fluctuations requires many-body techniques, like numerical renormalization group ${ }^{5,6}$ which are often applied to oversimplified models, like the single-orbital Anderson impurity model, although there are recent attempts to join together the two approaches $\underline{\underline{7}-\underline{9}}$ Unfortunately, out of equilibrium properties are much more difficult to study. Apart from many-body Keldish perturbation theory, ${ }^{10}$ many sophisticated numerical techniques have been developed in recent years to cope simultaneously with out-of-equilibrium and strong correlations $\stackrel{11-16}{1}$ However, given the complexity of the electronic structure that may arise at a nanocontact e.g. of a molecule or a bridging transition metal atom, it would be desirable to have at disposal approximate techniques simple and flexible enough to deal with realistic situations otherwise prohibitive with more accurate numerical approaches, as those previously mentioned.

In this paper we shall propose an out-of-equilibrium extension of the conventional variational approach, and, on such basis, an out-of-equilibrium extension of the Gutzwiller approximation 17,18 for correlated electron systems.

The paper is organized as follows. In Sec. II we briefly introduce the Hershfield formulation of the nonequilibrium steady state problem in quantum dots. In Sec. III we introduce the concept of scattering operators and derive some results related with the resonant model. In Sec. IV and $\mathrm{V}$ we derive the variational principle that defines the nonequilibrium steady state transport across a finite junction (e.g. a quantum dot) coupled to biased infinite leads. In Sec. VI we formulate a Fermi-liquid assumption for the system in the low energy/temperature/bias regime. In Sec. VII we introduce very briefly the standard Gutzwiller variational method for the single band Anderson impurity model in equilibrium. In Sec. VIII we propose a generalization of the Gutzwiller variational method to nonequilibrium. Finally, Sec. IX is devoted to the conclusions.

\section{THE PROBLEM}

We consider two biased macroscopic leads described by non-interacting electrons coupled to a bridging region, the quantum dot, described by discrete electronic multiplets

$$
\hat{\mathcal{H}}=\hat{T}+\hat{V}+\hat{\mathcal{H}}_{i n t},
$$

where $\hat{V}$ describes the tunnelling between the leads and the nanocontact and $\hat{\mathcal{H}}_{\text {int }}$ the local interaction in the nanocontact.

One assumes that initially the leads are not coupled through the bridging region, each lead being subject to a different electrochemical potential. Such a situation can be described by a density matrix

$$
\rho_{0}=e^{-\beta \hat{\mathcal{H}}_{0}(\Phi)} / \operatorname{Tr}\left(e^{-\beta \hat{\mathcal{H}}_{0}(\Phi)}\right)
$$

where

$$
\hat{\mathcal{H}}_{0}(\Phi)=\hat{\mathcal{H}}_{0}+\Phi \hat{Y}_{0}
$$


with $\mathcal{H}_{0}$ the non-interacting Hamiltonian of the independent left $(L)$ and right $(R)$ leads plus the nanocontact

$$
\hat{\mathcal{H}}_{0} \equiv \hat{T}+\hat{\mathcal{H}}_{i n t},
$$

$\Phi$ is the applied voltage between the two leads, and

$$
\Phi \hat{Y}_{0}=\Phi\left(\hat{N}_{L}-\hat{N}_{R}\right) / 2
$$

that describes the electrostatic energy gain due to the presence of the bias voltage, where $\hat{N}$ is the number operator - the initial state is stationary though out-ofequilibrium, equilibrium meant to be the two leads at the same chemical potential.

Suddenly the coupling to the bridging region is switched on - namely the Hamiltonian changes from $\hat{T}+\hat{\mathcal{H}}_{\text {int }}$ into $\hat{\mathcal{H}}=\hat{T}+\hat{\mathcal{H}}_{\text {int }}+\hat{V}$ - and a current starts to flow. If

$$
U(t)=e^{-i \hat{\mathcal{H}} t}
$$

is the time evolution operator with the full interaction, the initial density matrix $\rho_{0}$ evolves in time maintaining the functional form of a Boltzmann exponential

$$
\rho(t)=e^{-\beta \hat{\mathcal{H}}(t, \Phi)} / \operatorname{Tr}\left(e^{-\beta \hat{\mathcal{H}}(t, \Phi)}\right)
$$

where

$$
\hat{\mathcal{H}}(t, \Phi)=\hat{\mathcal{H}}(t)+\Phi \hat{Y}(t)
$$

and

$$
\begin{aligned}
\hat{\mathcal{H}}(t) & =U(t)\left(\hat{T}+\hat{\mathcal{H}}_{\text {int }}\right) U(t)^{\dagger}, \\
\hat{Y}(t) & =U(t) \hat{Y}_{0} U(t)^{\dagger}
\end{aligned}
$$

For time $t$ sufficiently large, namely after a transient time $\mathcal{T}$, the system reaches a steady state with constant current. If we are interested only in steady state properties, a good starting point is offered by Hershfield's results $\frac{19}{1}$ He showed that the stationary state value of certain observables coincide with their equilibrium value obtained through the effective density matrix

$$
\rho=e^{-\beta \hat{\mathcal{H}}(\Phi)} / \operatorname{Tr}\left(e^{-\beta \hat{\mathcal{H}}(\Phi)}\right),
$$

with

$$
\hat{\mathcal{H}}(\Phi)=\hat{\mathcal{H}}+\Phi \hat{Y}
$$

where $\hat{Y}$ is the asymptotic time evolution of $\hat{Y}_{0}$ still satisfying 28

$$
[\hat{\mathcal{H}}, \hat{Y}]=0
$$

Should $\hat{Y}$ be known, steady state properties could in principle be obtained by any equilibrium technique.

\section{THE RESONANT-MODEL OUT OF EQUILIBRIUM}

Let us consider the simple case of a non-interacting single-level quantum dot

$$
\hat{\mathcal{H}}_{0}=\hat{T}+\hat{V},
$$

with

$$
\begin{aligned}
& \hat{T}=\sum_{\alpha=-1,1} \sum_{k \sigma} \epsilon_{k} c_{\alpha k \sigma}^{\dagger} c_{\alpha k \sigma}+\sum_{\sigma} \epsilon_{d} d_{\sigma}^{\dagger} d_{\sigma}, \\
& \hat{V}=\sum_{\alpha=-1,1} \sum_{k \sigma} \frac{V_{k}}{\sqrt{\Omega}} d_{\sigma}^{\dagger} c_{\alpha k \sigma}+H . c .
\end{aligned}
$$

where $c_{\alpha k \sigma}^{\dagger}$ creates a conduction electron on the left $(\alpha=-1)$ or right $(\alpha=1)$ lead with quantum number $\mathrm{k}$ and spin $\sigma$ while $d_{\sigma}^{\dagger}$ creates an electron in the dot with spin $\sigma$, and $\Omega$ is the quantization volume of the system. Notice that, quite generally only a single channel of conduction electrons is coupled to the impurity, so that the model can always be mapped onto two one-dimensional leads hybridized at the contiguous edges with an impurity. Therefore it is perfectly legitimate to regard the quantum number $k$ as one-dimensional momentum and $\Omega$ as the linear size of the system.

Let us assume that our system does not have bound states. In this case it can be proven ${ }^{19,20}$ that the nonequilibrium Hamiltonian

$$
\hat{\mathcal{H}}_{0}(\Phi)=\hat{\mathcal{H}}_{0}+\Phi \hat{Y}_{0}
$$

can be expressed as

$$
\begin{aligned}
& \hat{\mathcal{H}}_{0}=\sum_{\alpha=-1,1} \sum_{k \sigma} \epsilon_{k} \psi_{\alpha k \sigma}^{\dagger} \psi_{\alpha k \sigma}, \\
& \hat{Y}_{0}=\sum_{\alpha=-1,1} \sum_{k \sigma} \frac{\alpha}{2} \psi_{\alpha k \sigma}^{\dagger} \psi_{\alpha k \sigma} ;
\end{aligned}
$$

where $\psi_{\alpha k \sigma}^{\dagger}$ are the fermionic creation operators that generate the left $(\alpha=-1)$ and right $(\alpha=1)$ incident scattering waves

$$
\begin{aligned}
\psi_{\alpha k \sigma}^{\dagger}|0\rangle & =\left[1+\frac{1}{\epsilon_{k}-\hat{\mathcal{H}}+i 0^{+}} \hat{V}\right] c_{\alpha k \sigma}^{\dagger}|0\rangle \\
& =c_{\alpha k \sigma}^{\dagger}|0\rangle+\frac{V_{k}}{\sqrt{\Omega}} g_{d}\left(\epsilon_{k}\right) d_{\sigma}^{\dagger}|0\rangle \\
& +\sum_{\alpha^{\prime} k^{\prime} \sigma^{\prime}} \frac{V_{k} V_{k^{\prime}}}{\Omega} \frac{g_{d}\left(\epsilon_{k}\right)}{\epsilon_{k}-\epsilon_{k^{\prime}}+i 0^{+}} c_{\alpha^{\prime} k^{\prime} \sigma^{\prime}}^{\dagger}|0\rangle
\end{aligned}
$$

being $g_{d}(\epsilon)$ the retarded Green's function of the impurity at equilibrium, which, in the infinite bandwidth limit, is given by

$$
g_{d}(\epsilon)=\frac{1}{\epsilon-\epsilon_{d}+i \Gamma} .
$$


We underline that Eq. (17) is meaningful only in the thermodynamic limit, i.e. when $\Omega \rightarrow \infty$. For a finite system the time evolution of an incident state

$$
\left|\psi_{\alpha k \sigma}^{i n}\right\rangle=c_{\alpha k \sigma}^{\dagger}|0\rangle
$$

oscillates, namely it doesn't converge to a well defined scattering state

$$
\left|\psi_{\alpha k \sigma}\right\rangle=\psi_{\alpha k \sigma}^{\dagger}|0\rangle
$$

The scattering states (17) constitute, in the thermodynamic limit, a complete basis

$$
\sum_{\alpha k \sigma} \psi_{\alpha k \sigma}^{\dagger} \psi_{\alpha k \sigma}=\sum_{\alpha k \sigma} c_{\alpha k \sigma}^{\dagger} c_{\alpha k \sigma}+\sum_{\sigma} d_{\sigma}^{\dagger} d_{\sigma},
$$

provided that there exist no bound states ${ }^{20}$ Eq. (21) allows us to formally expand the $c$ and $d$ operators as follows

$$
\begin{aligned}
c_{\bar{\alpha} \bar{k} \bar{\sigma}}^{\dagger} & =\psi_{\bar{\alpha} \bar{k} \bar{\sigma}}^{\dagger}+\sum_{\alpha k} \frac{V_{\bar{k}} V_{k}}{\Omega} \frac{g^{*}\left(\epsilon_{k}\right)}{\epsilon_{k}-\epsilon_{\bar{k}}-i 0^{+}} \psi_{\alpha k \bar{\sigma}}^{\dagger} \\
d_{\bar{\sigma}}^{\dagger} & =\sum_{\alpha k} \frac{V_{k}}{\sqrt{\Omega}} g^{*}\left(\epsilon_{k}\right) \psi_{\alpha k \bar{\sigma}}^{\dagger},
\end{aligned}
$$

and to calculate the average of any operator using the result

$$
\left\langle\Psi(\Phi)\left|\psi_{\alpha k}^{\dagger} \psi_{\alpha^{\prime} k^{\prime}}\right| \Psi(\Phi)\right\rangle=\delta_{\alpha \alpha^{\prime}} \delta_{k k^{\prime}} f\left(\epsilon_{k}+\Phi \frac{\alpha}{2}\right)
$$

- where $|\Psi(\Phi)\rangle$ is the ground state of $\hat{\mathcal{H}}_{0}(\Phi)$ and $f(\epsilon)$ is the Fermi function. The correct value of the average is finally obtained taking the limit for $\Omega \rightarrow \infty$ of the result. It can be proven that the obtained value is the same that one could obtain within the Keldish technique.

It is very important to underline that the scattering operators can formally be defined even in the interacting case, although their explicit calculation is not feasible in practice. If, for instance, we add to the resonant model Hamiltonian (13) a Hubbard repulsion term on the impurity

$$
\hat{U}=\frac{U}{2}\left(\hat{n}_{d}-1\right)^{2}
$$

(Anderson impurity model), the scattering operators $\psi_{\alpha k \sigma}^{\dagger}$ are defined as the asymptotic time evolution of the $c_{\alpha k \sigma}^{\dagger}$ operators generated by the full Anderson Hamiltonian

$$
\hat{\mathcal{H}}=\hat{T}+\hat{V}+\hat{U}
$$

and still satisfy the completeness relation (21) in the absence of bound states ${ }^{20}$ Moreover the interacting Hamiltonian (25) can still be expressed in terms of scattering $\operatorname{states}^{20}$

$$
\hat{\mathcal{H}}=\sum_{\alpha=-1,1} \sum_{k \sigma} \epsilon_{k} \psi_{\alpha k \sigma}^{\dagger} \psi_{\alpha k \sigma}
$$

$$
\hat{Y}=\sum_{\alpha=-1,1} \sum_{k \sigma} \frac{\alpha}{2} \psi_{\alpha k \sigma}^{\dagger} \psi_{\alpha k \sigma}
$$

We conclude this section calculating the energy $\mathcal{E}_{\Phi}$ for the non-interacting model (13). More precisely, we consider

$$
\delta \mathcal{E}_{\Phi}=\mathcal{E}_{\Phi}-\mathcal{E}_{\Phi}^{0},
$$

where $\mathcal{E}_{\Phi}^{0}$ is the energy of the uncorrelated system $\hat{T}$. Using Eq. (22) it can be proven that

$$
\begin{aligned}
\delta \mathcal{E}_{\Phi} & =-T \sum_{n, \alpha} \ln \left(\frac{i \epsilon_{n}+\alpha \frac{\Phi}{2}-\epsilon_{d}-\Delta\left(i \epsilon_{n}+\alpha \frac{\Phi}{2}\right)}{i \epsilon_{n}+\alpha \frac{\Phi}{2}-\epsilon_{d}}\right) \\
& =-\int \frac{d \epsilon}{\pi} \delta(\epsilon)\left[f\left(\epsilon+\frac{\Phi}{2}\right)+f\left(\epsilon-\frac{\Phi}{2}\right)\right] ;
\end{aligned}
$$

where $\epsilon_{n}$ are Matsubara frequencies,

$$
\Delta(z)=\frac{1}{\Omega} \sum_{k \alpha} \frac{V_{k}^{2}}{z-\epsilon_{k}}
$$

is the hybridization function, and

$$
\delta(\epsilon)=-\operatorname{Im} \ln \left(\frac{\epsilon+i 0^{+}-\epsilon_{d}-\Delta\left(\epsilon+i 0^{+}\right)}{\epsilon+i 0^{+}-\epsilon_{d}}\right) .
$$

If we assume that the half-bandwidth $W$ is the unit of energy, that the density of states is flat

$$
\begin{aligned}
\Delta(z) & =\int \frac{d \epsilon}{\pi} \frac{\Gamma(\epsilon)}{z-\epsilon} \\
\Gamma(\epsilon) & =\Gamma \chi_{[-1,1]}(\epsilon) \\
\chi_{[-1,1]}(\epsilon) & =\left\{\begin{array}{ll}
1 & \forall \epsilon \in[-1,1] \\
0 & \forall \epsilon \notin[-1,1]
\end{array},\right.
\end{aligned}
$$

and that $\Gamma \ll W=1$, it can be easily verified that

$$
\left.\Gamma^{2} \frac{\partial}{\partial \Gamma}\left(\frac{\delta \mathcal{E}_{\Phi}(\Gamma)}{\Gamma}\right)=-\frac{2}{\pi} \epsilon \arctan \left(\frac{\Gamma}{\epsilon}\right)\right]_{-1}^{-\frac{\Phi}{2}}
$$

We observe that when $\Phi=0$ the right member of Eq. (32) is $2 \Gamma / \pi$, so that the solution of Eq. (32) is

$$
\delta \mathcal{E}_{0}=-\frac{2}{\pi} \Gamma \log \left(\frac{e}{\Gamma}\right)
$$

(being $e$ the Nepero's number), which derives from an hybridization gain

$$
\delta \mathcal{E}_{h y b}=-\frac{4}{\pi} \Gamma \log \left(\frac{1}{\Gamma}\right)
$$

and a bath energy cost

$$
\delta \mathcal{E}_{\text {bath }}=\frac{2}{\pi} \Gamma \log \left(\frac{1}{e \Gamma}\right) .
$$

In the regime

$$
W=1 \gg \Phi \gg \Gamma,
$$

the right member of Eq. (32) vanishes, so that

$$
\delta \mathcal{E}_{\Phi}=\frac{2}{\pi} \Gamma \log \left(\frac{\Phi}{2}\right) .
$$




\section{THE BIAS AS AN "ORDER PARAMETER"}

In this section we propose a point of view of the nonequilibrium problem in quantum dots based on ideas and definitions very similar to those encountered in the general theoretical description of collective phenomena in quantum mechanics. 21

In the standard quantum theory of finite systems there is a one-to-one correspondence between the observables and the operators in a certain Hilbert space. This correspondence is unique (Von Neumann 1955), and this ensures that the choice of a specific representation of a finite system does not lead to loss of generality. The situation is different for infinite systems, because Von Neumann's theorem can no longer be applied. The observable of an infinite systems generally admit a big variety of inequivalent representations, corresponding to macroscopically different classes of states.

Let us consider, for instance, the case of the Heisenberg model

$$
\hat{\mathcal{H}}=-J \sum_{n} \mathbf{S}_{n} \mathbf{S}_{n+1}, \quad J>0 .
$$

A ground state $|\Psi\rangle$ of the system has all the spins aligned parallel in the same direction. The class of all the states obtained applying a finite number of local spin transformations to $|\Psi\rangle$ does not change its magnetic order parameter

$$
\mathbf{m}=\lim _{N \rightarrow \infty} \sum_{n=-N}^{N} \frac{\left\langle\Psi\left|\mathbf{S}_{n}\right| \Psi\right\rangle}{2 N+1}
$$

because the system is infinite. Furthermore the topological closure of the space spanned by these states (that is a Hilbert space) is the basis of an irreducible representation of the algebra of the Pauli spins $\left\{\mathbf{S}_{n}\right\}$. In this example we have a set of different phases corresponding to different (inequivalent) representations of the observables for any arbitrary direction $\mathbf{m}$. In other words, a state $\left|\Psi_{\mathbf{m}}\right\rangle$ defines a corresponding island of states $\mathcal{I}\left(\Psi_{\mathbf{m}}\right)$ that share the same order parameter and are the basis of a specific representation of the algebra of the observables.

The general formal definition of the islands is based on the fundamental Gelfand-Naimark-Segal (GNS) theorem, ${ }^{21}$ that tells us that if we have an algebra $\mathcal{A}$ generated by the local observables of an infinite system - i.e. all the operators that belong to any finite region of space $\Lambda$, - for each state $\rho$ there is an operator-representation $\hat{\mathcal{A}}_{\rho}$ of $\mathcal{A}$ on a Hilbert space $\mathcal{H}_{\rho}$, which is determined (up to unitary equivalence) by the conditions that it exists a vector $\left|\Psi_{\rho}\right\rangle$ in $\mathcal{H}_{\rho}$ such that

$$
\rho(A)=\left\langle\Psi_{\rho}|\hat{A}| \Psi_{\rho}\right\rangle \quad \forall A \in \mathcal{A},
$$

and that $\mathcal{H}_{\rho}$ is generated by applying the elements of $\hat{\mathcal{A}}_{\rho}$ to $\left|\Psi_{\rho}\right\rangle$.

The island $\mathcal{I}(\rho)$ is, by definition, the set of all the states $\rho^{\prime}$ corresponding to all the density matrices $\hat{\rho}^{\prime}$ in $\mathcal{H}_{\rho}$.
Physically, the meaning of the states $\rho^{\prime} \in \mathcal{I}(\rho)$ is that these are states generated by localized modifications of $\rho$, but are "macroscopically" equivalent to one other.

Let us now consider the system represented in Fig. 1, whose dynamics is defined by the interacting Anderson impurity model

$$
\begin{aligned}
\hat{\mathcal{H}} & =\sum_{\alpha k \sigma} \epsilon_{k} c_{\alpha k \sigma}^{\dagger} c_{\alpha k \sigma}+\sum_{\alpha k \sigma} \frac{V_{k}}{\sqrt{\Omega}} d_{\sigma}^{\dagger} c_{\alpha k \sigma}+H . c . \\
& +\sum_{\sigma} \epsilon_{d} d_{\sigma}^{\dagger} d_{\sigma}+\frac{U}{2}\left(\hat{n}_{d}-1\right)^{2},
\end{aligned}
$$

where

$$
\hat{n}_{d}=\sum_{\sigma} d_{\sigma}^{\dagger} d_{\sigma}
$$

is the impurity number operator.

To the initial state $\rho_{0}(\Phi, T)$ defined in Eq. (2) will correspond, through the GNS theorem, an island of states

$$
\mathcal{I}(\Phi, T) \equiv \mathcal{I}\left(\rho_{0}(\Phi, T)\right),
$$

and the islands obtained from initial states with different $\Phi$ will correspond to inequivalent phases, because of the infinite volume of the two leads. In this sense we can say that $\Phi$ plays the same role as the magnetic order parameter in the example of the Heisenberg model considered above.

In this work we assume that the dynamics of the system does not mix vectors belonging to different phases, i.e. that if $\rho^{\prime}$ belongs to $\mathcal{I}(\Phi, T)$ then so does $\rho^{\prime}(t)$. This corresponds to the physical idea that the system never equilibrates because the two leads are infinite and the junction between them (the dot) is finite, so that the current through the dot can not change the densities of the two leads defined by the value of $\Phi$.

The stability of the dynamics in $\mathcal{I}(\Phi, T)$ allows us to consider $\hat{\mathcal{H}}_{\Phi, T}$ the generator of the time evolution transformation in $\mathcal{I}(\Phi, T)$, i.e. the Hamiltonian that describes the dynamics of the island.

The basis of the ideas proposed in this paper is that an equilibrium problem corresponds to the study of the $\Phi=$ 0 phase, while a nonequilibrium problem is equivalent to study a finite- $\Phi$ phase. Once the $\hat{\mathcal{H}}_{\Phi, T}$ operator is defined we can apply, in principle, any equilibrium technique to study the physics of the corresponding island.

\section{VARIATIONAL APPROACH AT $T=0$}

In this section we will concentrate our attention to a general GNS representation of the system at $T=0$

$$
\mathcal{I}(\Phi) \equiv \mathcal{I}(\Phi, T=0) .
$$

In this case we know that the initial state is represented by a pure vector

$$
\left|\Psi_{0}(\Phi)\right\rangle \in \mathcal{I}(\Phi)
$$




$$
\rho_{0}(\Phi, T=0)=\left|\Psi_{0}(\Phi)\right\rangle\left\langle\Psi_{0}(\Phi)\right|,
$$

and that the operator that governs the dynamics of $\mathcal{I}(\Phi)$ is simply given by

$$
\begin{aligned}
& \hat{\mathcal{H}}_{\Phi} \equiv \hat{\mathcal{H}}_{\Phi, T=0} \\
& \hat{\mathcal{H}}_{\Phi}=P_{\Phi} \hat{\mathcal{H}} P_{\Phi},
\end{aligned}
$$

where $P_{\Phi}$ is the projector on $\mathcal{I}(\Phi)$.

We define $\overline{\mathcal{I}}(\Phi)$ as the product of the Hilbert space of the dot and the Hilbert space generated by $\left|\Psi_{0}(\Phi)\right\rangle$ and all the states

$$
\left|\Psi_{0 S}\right\rangle=\prod_{(\alpha, k, \sigma) \in S} c_{\alpha k \sigma}^{\dagger}|0\rangle
$$

where $S$ is any subset of

$$
E=\{(\alpha, k, \sigma) \mid \alpha= \pm 1, k \in[-\pi, \pi], \sigma= \pm 1 / 2\}
$$

that differs from the set $S_{\Phi}$ of the occupied states of

$$
\left|\Psi_{0}(\Phi)\right\rangle \equiv \prod_{(\alpha, k, \sigma) \in S_{\Phi}} c_{\alpha k \sigma}^{\dagger}|0\rangle .
$$

by an arbitrary, but finite, number of particle-hole transformations. In other words, any state $\left|\Psi_{0 S}\right\rangle$ can be written in the form

$$
\left|\Psi_{0 S}\right\rangle=\prod_{(\alpha, k, \sigma) \in \mathcal{K}_{S}} \prod_{\left(\alpha^{\prime}, k^{\prime} \sigma^{\prime}\right) \in \mathcal{K}_{S}^{\prime}} c_{\alpha k \sigma}^{\dagger} c_{\alpha^{\prime} k^{\prime} \sigma^{\prime}}\left|\Psi_{0}(\Phi)\right\rangle,
$$

where $\mathcal{K}_{S}$ and $\mathcal{K}_{S}^{\prime}$ are finite subsets of $E$.

We are going to prove that $\mathcal{I}(\Phi)$ coincides with $\overline{\mathcal{I}}(\Phi)$. To prove that $\overline{\mathcal{I}}(\Phi) \subset \mathcal{I}(\Phi)$ we observe that whether a state $|\Psi\rangle \in \overline{\mathcal{I}}(\Phi)$ belongs to $\mathcal{I}(\Phi)$ also the state

$$
\left|\Psi_{\alpha k \sigma, \alpha^{\prime} k^{\prime} \sigma^{\prime}}^{\Lambda}\right\rangle \equiv \sum_{R R^{\prime} \in \Lambda} e^{i k R} e^{-i k^{\prime} R^{\prime}} c_{\alpha R \sigma}^{\dagger} c_{\alpha^{\prime} R^{\prime} \sigma^{\prime}}|\Psi\rangle
$$

belongs to it for any finite region of space $\Lambda$. But, by definition, $\mathcal{I}(\Phi)$ is the topological closure of the space generated by the states obtained modifying $\left|\Psi_{0}(\Phi)\right\rangle$ locally, so that the state

$$
c_{\alpha k \sigma}^{\dagger} c_{\alpha^{\prime} k^{\prime} \sigma^{\prime}}|\Psi\rangle=\lim _{\Lambda \uparrow}\left|\Psi_{\alpha k \sigma, \alpha^{\prime} k^{\prime} \sigma^{\prime}}^{\Lambda}\right\rangle
$$

where $\lim _{\Lambda \uparrow}$ denotes the limit for the size $|\Lambda|$ of $\Lambda$ going to infinity, belongs to $\mathcal{I}(\Phi)$ too. The inclusion $\overline{\mathcal{I}}(\Phi) \subset \mathcal{I}(\Phi)$ is then proven by induction.

To prove that $\mathcal{I}(\Phi) \subset \overline{\mathcal{I}}(\Phi)$ we consider again the set $E$ defined in Eq. (48) of all the possible values of $(\alpha, k, \sigma)$, and we associate to each subset $e \subset E$ the operator

$$
N_{e}^{\Lambda}=\sum_{\left(\alpha, k=\frac{2 \pi}{|\Lambda|} n, \sigma\right) \in e} c_{\alpha, k, \sigma}^{\dagger} c_{\alpha, k, \sigma} \quad(n \text { integer }) .
$$

Let us consider a general state $|\Psi\rangle \in \mathcal{I}(\Phi)$. If $|\Psi\rangle$ does not belong to $\overline{\mathcal{I}}(\Phi)$ then, by definition, one can define a set $e \subset E$ of single particle states such that either

$$
\lim _{\Lambda \uparrow} \frac{1}{|\Lambda|}\left\langle\Psi\left|N_{e}^{\Lambda}\right| \Psi\right\rangle>0
$$

$$
\lim _{\Lambda \uparrow} \frac{1}{|\Lambda|}\left\langle\Psi_{0}(\Phi)\left|N_{e}^{\Lambda}\right| \Psi_{0}(\Phi)\right\rangle=0
$$

or

$$
\begin{array}{r}
\lim _{\Lambda \uparrow} \frac{1}{|\Lambda|}\left\langle\Psi_{0}(\Phi)\left|N_{e}^{\Lambda}\right| \Psi_{0}(\Phi)\right\rangle>0 \\
\lim _{\Lambda \uparrow} \frac{1}{|\Lambda|}\left\langle\Psi\left|N_{e}^{\Lambda}\right| \Psi\right\rangle=0,
\end{array}
$$

But, if such set exists, it is clear that $|\Psi\rangle$ can't be generated by the application of local observables to $\left|\Psi_{0}(\Phi)\right\rangle$, because the contribution of local modifications to Eqs. (54]55) vanishes in the limit of $|\Lambda| \rightarrow \infty$. The inclusion $\mathcal{I}(\Phi) \subset \overline{\mathcal{I}}(\Phi)$ is then proven.

We can reformulate the statement $\mathcal{I}(\Phi)=\overline{\mathcal{I}}(\Phi)$ saying that the measure $d \mu_{\Psi}(\alpha, k, \sigma)$ such that

$$
\int_{e} d \mu_{\Psi}(\alpha, k, \sigma) \equiv \lim _{\Lambda \uparrow} \frac{1}{|\Lambda|}\left\langle\Psi\left|N_{e}^{\Lambda}\right| \Psi\right\rangle \quad \forall e \subset E
$$

is an order parameter that identifies the phase $\mathcal{I}(\Phi)$; i.e. that the states $|\Psi\rangle$ that belong to $\mathcal{I}(\Phi)$ are characterized by the condition

$$
d \mu_{\Psi}(\alpha, k, \sigma)=d \mu_{\Psi_{0}(\Phi)}(\alpha, k, \sigma) .
$$

We underline the strong analogy between the measure $d \mu_{\Psi}(\alpha, k, \sigma)$ and the magnetic order parameter $\mathbf{m}$, see Eq. (39), for the Heisenberg model.

As we have anticipated in the previous section, we assume that $\mathcal{I}(\Phi)$ is stable respect to the dynamics induced by the dot. While such assumption is very reasonable for finite time evolutions, it is less trivial that the steady state, which is reached only after an infinite time, still belongs to $\mathcal{I}(\Phi)$ - and this is what we need. Although we can't prove the stability of the $\mathcal{I}(\Phi)$ respect to the asymptotic dynamic induced by an interacting dot, it is encouraging to observe that when $U=0$

$$
d \mu_{\Psi(\Phi)}(\alpha, k, \sigma)=d \mu_{\Psi_{0}(\Phi)}(\alpha, k, \sigma) .
$$

This can be easily verified from the following equation

$$
|\Psi(\Phi)\rangle \equiv \prod_{(\alpha, k, \sigma) \in S_{\Phi}} \psi_{\alpha k \sigma}^{\dagger}|0\rangle,
$$

where the scattering operators $\psi_{\alpha k \sigma}^{\dagger}$ are given by Eq. (17) and $S_{\Phi} \subset E$ is defined by Eq (49).

As a consequence of the stability of the (asymptotic) dynamics induced by the dot we can characterize $\mathcal{I}(\Phi)$, in the absence of bound states (when Eq. (21) is satisfied $), 20$ even as the space generated by $|\Psi(\Phi)\rangle$ and all the asymptotic time evolutions of the eigenstates of $\hat{\mathcal{H}}_{0}$ defined in Eq. (47), i.e. the states

$$
\left|\Psi_{S}\right\rangle=\prod_{(\alpha, k, \sigma) \in S} \psi_{\alpha k \sigma}^{\dagger}|0\rangle
$$

where $S$ differs from the set $S_{\Phi}$ of the occupied states of $|\Psi(\Phi)\rangle$ by an arbitrary, but finite, number of differences, 
and $\psi_{\alpha k \sigma}^{\dagger}$ are the scattering operators of Eq. (26) (that are interacting in general).

Starting from the above characterization of $\mathcal{I}(\Phi)$ we can understand that all the possible eigenstates $\left|\Psi_{S}\right\rangle$ of $\hat{\mathcal{H}}$ defined in $\mathcal{I}(\Phi)$ - i.e. the eigenstates of $\hat{\mathcal{H}}_{\Phi}$ - correspond to the same current

$$
I_{S}=-i \sum_{k \sigma} \frac{V_{k}}{\sqrt{\Omega}}\left\langle\Psi(\Phi)\left|d_{\sigma}^{\dagger} c_{k \sigma,-1}\right| \Psi(\Phi)\right\rangle-c . c .
$$

and can be considered "equivalent" in this sense. Any of the eigenstates $\left|\Psi_{S}\right\rangle$ of $\hat{\mathcal{H}}$ in $\mathcal{I}(\Phi)$ defined in Eq. (60). is, in fact, the asymptotic time evolution of the corresponding "initial" state

$$
\left|\Psi_{0 S}\right\rangle \equiv \prod_{(\alpha, k, \sigma) \in S} c_{\alpha k \sigma}^{\dagger}|0\rangle
$$

The existence of a particular set $\bar{S}$ such that

$$
I_{\bar{S}} \neq I \equiv\langle\Psi(\Phi)|\hat{I}| \Psi(\Phi)\rangle
$$

would imply that the state $\left|\Psi_{0 \bar{S}}\right\rangle$ - obtained, by definition, modifying the initial state $\left|\Psi_{0}(\Phi)\right\rangle$ applying to it only a finite number of particle-hole transformation leads, after an infinite transient time, to a different current respect to the one of the steady state $|\Psi(\Phi)\rangle$, and this is clearly unphysical (notice that the same argument can be applied to any local observable, and not only to the current operator).

It is interesting to check directly the validity of the above statement for the simple non-interacting case $U=$ 0 . The scattering operators are, in this case, given by Eq. (17), and the average of the current operator can be calculated with Eq. (22) and the identity

$$
\left\langle\Psi_{S}\left|\psi_{\alpha k \sigma}^{\dagger} \psi_{\alpha^{\prime} k^{\prime} \sigma^{\prime}}\right| \Psi_{S}\right\rangle=\delta_{\alpha \alpha^{\prime}} \delta_{k k^{\prime}} \delta_{\sigma \sigma^{\prime}} n_{\Psi_{S}}(\alpha, k, \sigma) ;
$$

where

$$
n_{\Psi_{S}}(\alpha, k, \sigma)= \begin{cases}1 & \text { if }(\alpha, k, \sigma) \in S[\Psi] \\ 0 & \text { if }(\alpha, k, \sigma) \notin S[\Psi] .\end{cases}
$$

The result is

$$
I=\int d \epsilon \Gamma(\epsilon) \rho_{d}(\epsilon) \sum_{\alpha \sigma} \alpha n_{\Psi_{S}}(\alpha, \epsilon, \sigma),
$$

where $\rho_{d}(\epsilon)$ is the spectral function of the dot

$$
\rho_{d}(\epsilon)=-\frac{1}{\pi} \operatorname{Im}\left(g_{d}\left(\epsilon+i 0^{+}\right)\right) .
$$

If $|\Psi\rangle \in \mathcal{I}(\Phi)=\overline{\mathcal{I}}(\Phi)$ then

$$
n_{\Psi_{S}}(\alpha, \epsilon, \sigma) \simeq f\left(\epsilon+\Phi \frac{\alpha}{2}\right),
$$

i.e. the difference between the two functions does not contribute to the integral (66) that defines the value of the current $I$, because, by definition,

$$
\sum_{\alpha \sigma} \int d \epsilon\left|n_{\Psi_{S}}(\alpha, \epsilon, \sigma)-f\left(\epsilon+\Phi \frac{\alpha}{2}\right)\right|=0 .
$$

In order to identify the eigenstates of $\hat{\mathcal{H}}_{\Phi}$ a possibility is to minimize the variance

$$
\begin{aligned}
\sigma_{\Phi}^{2}\left[\Omega_{+}^{G}\right]= & \frac{\left\langle\Psi_{0}(\Phi)\left|\Omega_{+}^{G \dagger} \hat{\mathcal{H}}_{\Phi}^{2} \Omega_{+}^{G}\right| \Psi_{0}(\Phi)\right\rangle}{\left\langle\Psi_{0}(\Phi)\left|\Omega_{+}^{G \dagger} \Omega_{+}^{G}\right| \Psi_{0}(\Phi)\right\rangle} \\
& -\left(\frac{\left\langle\Psi_{0}(\Phi)\left|\Omega_{+}^{G \dagger} \hat{\mathcal{H}}_{\Phi} \Omega_{+}^{G}\right| \Psi_{0}(\Phi)\right\rangle}{\left\langle\Psi_{0}(\Phi)\left|\Omega_{+}^{G \dagger} \Omega_{+}^{G}\right| \Psi_{0}(\Phi)\right\rangle}\right)^{2}
\end{aligned}
$$

respect to the most general operator $\Omega_{+}^{G}$ that is generated by the algebra of the local observables. Notice that what we have defined is a variational principle for the Hershfield steady state at zero temperature!

\section{A. An energy-based approach}

One may be tempted to use the energy instead of the variance, i.e. to claim that the ground state can be calculated variationally even minimizing the energy

$$
\mathcal{E}_{\Phi}\left[\Omega_{+}^{G}\right]=\frac{\left\langle\Psi_{0}(\Phi)\left|\Omega_{+}^{G \dagger} \hat{\mathcal{H}}_{\Phi} \Omega_{+}^{G}\right| \Psi_{0}(\Phi)\right\rangle}{\left\langle\Psi_{0}(\Phi)\left|\Omega_{+}^{G \dagger} \Omega_{+}^{G}\right| \Psi_{0}(\Phi)\right\rangle} .
$$

respect to the $\Omega_{+}^{G}$-operators defined above.

Unfortunately such energy minimum does not exist, because a $\Phi>0$ phase contains states obtained from the Hershfield state $|\Psi(\Phi)\rangle$ moving an arbitrary (although finite) number of electrons from one of the leads to the other. A solution to this problem would be to consider only the limited subset of $\Omega_{+}^{G}$ such that $\Omega_{+}^{G}\left|\Psi_{0}(\Phi)\right\rangle$ satisfy the equation

$$
\hat{Y} \Omega_{+}^{G}\left|\Psi_{0}(\Phi)\right\rangle=\delta N_{0}(\Phi) \Omega_{+}^{G}\left|\Psi_{0}(\Phi)\right\rangle
$$

where

$$
\hat{Y}=\sum_{\alpha=-1,1} \sum_{k \sigma} \frac{\alpha}{2} \psi_{\alpha k \sigma}^{\dagger} \psi_{\alpha k \sigma}
$$

and $\delta N_{0}(\Phi)$ is defined by

$$
\hat{Y}_{0}\left|\Psi_{0}(\Phi)\right\rangle=\delta N_{0}(\Phi)\left|\Psi_{0}(\Phi)\right\rangle
$$

where

$$
\hat{Y}_{0}=\sum_{\alpha=-1,1} \sum_{k \sigma} \frac{\alpha}{2} c_{\alpha k \sigma}^{\dagger} c_{\alpha k \sigma} .
$$

In this subspace it is clear, if we think in terms of interacting scattering operators, that the only eigenstate of $\hat{\mathcal{H}}_{\Phi}$ is the state that minimizes the energy. Unfortunately the operator $\hat{Y}$ is not known, so that the energybased procedure defined here can't be applied rigorously in practice.

Notice that if we formally apply the exact (unknown) asymptotic time evolution operator $\Omega_{+}$to the two members of Eq. (74)

$$
\Omega_{+} \hat{Y}_{0}\left|\Psi_{0}(\Phi)\right\rangle=\delta N_{0}(\Phi) \Omega_{+}\left|\Psi_{0}(\Phi)\right\rangle
$$


we obtain exactly the condition defined in Eq. (72), namely that

$$
\begin{aligned}
\hat{Y} \Omega_{+}\left|\Psi_{0}(\Phi)\right\rangle & =\left(\Omega_{+} \hat{Y}_{0} \Omega_{+}^{\dagger}\right) \Omega_{+}\left|\Psi_{0}(\Phi)\right\rangle \\
& =\delta N_{0}(\Phi) \Omega_{+}\left|\Psi_{0}(\Phi)\right\rangle,
\end{aligned}
$$

where we have used that

$$
\hat{Y}=\Omega_{+} \hat{Y}_{0} \Omega_{+}^{\dagger} .
$$

If we apply the approximated trial $\Omega_{+}^{G}$ to Eq. (174) we obtain, instead, that

$$
\Omega_{+}^{G} \hat{Y}_{0}\left|\Psi_{0}(\Phi)\right\rangle=\delta N_{0}(\Phi) \Omega_{+}^{G}\left|\Psi_{0}(\Phi)\right\rangle,
$$

which is equivalent to

$$
\hat{Y}_{G} \Omega_{+}^{G}\left|\Psi_{0}(\Phi)\right\rangle=\delta N_{0}(\Phi) \Omega_{+}^{G}\left|\Psi_{0}(\Phi)\right\rangle,
$$

where

$$
\hat{Y}_{G}=\Omega_{+}^{G} \hat{Y}_{0} \Omega_{+}^{G-1} .
$$

For this reason, whether we believe, for some physical reason, that the proposed $\Omega_{+}^{G}$ is sufficiently good to guarantee (approximately) the equivalence of Eq. (80) and Eq. (72) the energy minimization procedure is still meaningful, although not purely variational.

In the following sections we will propose an example of such energy-based approach starting from a particular variational space, that is expected to describe sufficiently well the qualitative behaviour, in the FermiLiquid regime, of the interacting single-orbital Anderson impurity model.

We conclude this section observing that, at least formally, the GNS theorem mentioned above allows us to define a variational principle for the Hershfield steady state even at finite temperature. In fact, to a thermal state $\rho_{0}(\Phi, T)$ corresponds, in its GNS representation, a vector $\left|\Psi_{0}(\Phi, T)\right\rangle$, and the dynamics of the corresponding island $\mathcal{I}(\Phi, T)$ is (presumably) still generated by some operator $\hat{\mathcal{H}}_{\Phi, T}$, although it is not simply a "block" of $\hat{\mathcal{H}}$ (Eq. (46)). An eigenstate of $\hat{\mathcal{H}}_{\Phi, T}$ is a stationary state, i.e. the representation of the Hershfield state in $\mathcal{I}(\Phi, T)$, and can be formally identified by the minimum-variance condition as before.

\section{THE CONCEPT OF QUASI-PARTICLES OUT OF EQUILIBRIUM}

Let us consider again the general interacting system described by the Hamiltonian

$$
\hat{\mathcal{H}}=\hat{\mathcal{H}}_{0}+\hat{V}+\hat{\mathcal{H}}_{\text {int }},
$$

We know that if we prepare the two leads at a different chemical potentials and let it evolve within the interacting Hamiltonian

$$
U(t)=e^{-i \hat{\mathcal{H}} t}
$$

for times $t$ longer than some transient time $\mathcal{T}$ the final nonequilibrium state is described by the Hershfield Hamiltonian

$$
\hat{\mathcal{H}}(\Phi)=\hat{\mathcal{H}}+\Phi \hat{Y}
$$

formally defined in Eq. (9)

In general $\hat{Y}$ is a complicated many body operator that must satisfy Eq. (12) and in addition share the same symmetry properties as $\hat{Y}_{0}$, i.e. a spin-singlet operator odd under interchanging the two leads. Therefore, generally the steady-state Hamiltonian $\hat{\mathcal{H}}(\Phi)$ is an interacting one, the interaction

$$
\delta \mathcal{H}(\Phi) \equiv \hat{\mathcal{H}}_{i n t}+\Phi\left(\hat{Y}-\hat{Y}_{0}\right)
$$

presumably remaining "local" (in the sense defined in section (V) as it was originally. Furthermore, since the nanocontact can not change the bulk properties of the leads, e.g. inducing a spontaneous symmetry breaking, $\hat{\mathcal{H}}(\Phi)$ should still describe a metal. It is therefore tempting to assume that, if in the absence of external bias the system, leads plus nanocontact, is described by a local Fermi liquid theory in the Nozières sense, 22 which is generally the case, the same should hold even in the steady state after the bias is applied. 23 It then follows that it should be possible to represent the low energy/temperature/bias properties in terms of weakly interacting quasi-particles which, by continuity with the non-interacting case, should be better regarded as renormalized scattering states with an Hamiltonian of the same form as (16) with renormalized (bias dependent) energies plus additional weak local-interaction terms. ${ }^{22}$ This local Fermi-liquid assumption seems to us quite plausible. However, since the bias is coupled to a non-conserved quantity, the charge difference between the leads, the effective bias felt by the quasi-particles will generally differ from the applied one and the quasiparticle current does not correspond to the real one. This implies that the current can not be expressed simply in terms of Landau parameters and an explicit calculation is required.

\section{THE GUTZWILLER APPROXIMATION AT EQUILIBRIUM}

Let us consider the $\Phi=0$ phase, namely the equilibrium problem. Although the method we shall present is quite general, for sake of simplicity we shall show how it works in the simple case of a bridging region described by a single-orbital Anderson impurity model at half-filling

$$
\begin{aligned}
\hat{\mathcal{H}} & =\sum_{\alpha k \sigma} \epsilon_{k} c_{\alpha k \sigma}^{\dagger} c_{\alpha k \sigma}+\sum_{\alpha k \sigma} \frac{V_{k}}{\sqrt{\Omega}}\left(d_{\sigma}^{\dagger} c_{\alpha k \sigma}+H . c .\right) \\
& +\frac{U}{2}\left(\hat{n}_{d}-1\right)^{2} \equiv(\hat{T}+\hat{V})+\hat{U} \equiv \hat{\mathcal{H}}_{0}+\hat{U}
\end{aligned}
$$




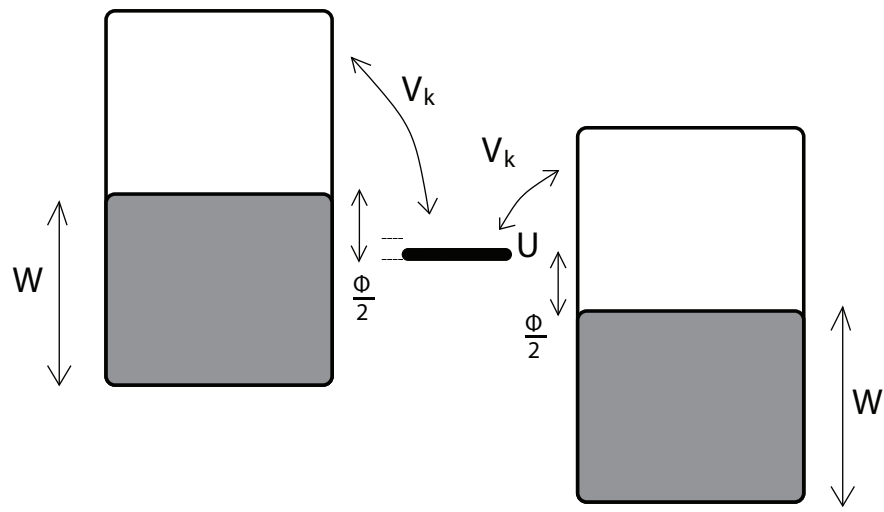

FIG. 1: (Color online) The single dot system.

The physical properties of the above Anderson impurity model are very well known ${ }^{24}$ For large $U$ the model effectively maps into a Kondo model, the impurity electron behaving as a local moment Kondo screened by the conduction electrons. A simple way to describe qualitatively and to some extent also quantitatively the Kondo screening is by a Gutzwiller-type of variational wavefunction 25,26

$$
|\Psi\rangle=\mathcal{P}_{d}\left|\Psi_{0}\right\rangle
$$

where $\mathcal{P}_{d}$ is an operator that modifies the relative weights of the impurity electronic configurations with respect to the uncorrelated wavefunction $\left|\Psi_{0}\right\rangle$

$$
\mathcal{P}_{d}=\lambda_{0,2}(|0\rangle\langle 0|+| \uparrow \downarrow\rangle\langle\uparrow \downarrow|)
$$

$$
+\lambda_{1}(|\uparrow\rangle\langle\uparrow|+| \downarrow\rangle\langle\downarrow|)
$$

and $\left|\Psi_{0}\right\rangle$ is the ground state of a non-interacting variational resonant level Hamiltonian.

\section{A. Energy optimization}

The variational procedure amounts to optimize both the local projector $\mathcal{P}_{d}$ as well as the non-interacting wavefunction $\left|\Psi_{0}\right\rangle$ by minimizing the expectation value of the Hamiltonian (86).

We assume that $\mathcal{P}_{d}$ is subject to the following two conditions

$$
\begin{aligned}
\left\langle\Psi_{0}\left|\mathcal{P}_{d}^{\dagger} \mathcal{P}_{d}\right| \Psi_{0}\right\rangle & =1 \\
\left\langle\Psi_{0}\left|\mathcal{P}_{d}^{\dagger} \mathcal{P}_{d} \hat{n}_{d \sigma}\right| \Psi_{0}\right\rangle & =\left\langle\Psi_{0}\left|\hat{n}_{d \sigma}\right| \Psi_{0}\right\rangle
\end{aligned}
$$

where

$$
\hat{n}_{d \sigma}=d_{\sigma}^{\dagger} d_{\sigma}
$$

Condition (89) is the normalization requirement of the variational wavefunction, that corresponds, in terms of $\lambda$-parameters, to the condition

$$
\lambda_{0,2}^{2}+\lambda_{1}^{2}=2 .
$$

Condition (90) - that ensures that all the Wick contractions between the conduction electron operators and the impurity operators are zero - allows to evaluate expectation values straightforwardly.

In particular, the expectation value of the Hamiltonian (86), that has to be minimized, is

$$
\begin{aligned}
E[\Psi] & =\frac{\langle\Psi|\hat{\mathcal{H}}| \Psi\rangle}{\langle\Psi \mid \Psi\rangle} \\
& =\left\langle\Psi_{0}\left|\left[\sum_{\alpha k \sigma} \epsilon_{k} c_{\alpha k \sigma}^{\dagger} c_{\alpha k \sigma}+\sum_{\alpha k \sigma} \frac{R V_{k}}{\sqrt{\Omega}}\left(d_{\sigma}^{\dagger} c_{\alpha k \sigma}+H . c .\right)\right]\right| \Psi_{0}\right\rangle+\frac{U}{2}\left\langle\Psi_{0}\left|\mathcal{P}_{d}^{\dagger}\left(\hat{n}_{d}-1\right)^{2} \mathcal{P}_{d}\right| \Psi_{0}\right\rangle \\
& \equiv\left\langle\Psi_{0}\left|\hat{\mathcal{H}}_{R}^{0}\right| \Psi_{0}\right\rangle+\frac{U}{2}\left\langle\Psi_{0}\left|\mathcal{P}_{d}^{\dagger}\left(\hat{n}_{d}-1\right)^{2} \mathcal{P}_{d}\right| \Psi_{0}\right\rangle
\end{aligned}
$$

where the hopping renormalization coefficient $R$ is obtained through the following equation:

$$
\left\langle\Psi_{0}\left|\mathcal{P}_{d}^{\dagger} d_{\sigma}^{\dagger} \mathcal{P}_{d} d_{\sigma}\right| \Psi_{0}\right\rangle=R\left\langle\Psi_{0}\left|d_{\sigma}^{\dagger} d_{\sigma}\right| \Psi_{0}\right\rangle,
$$

whose solution is

$$
R=\lambda_{0,2} \lambda_{1}
$$

The calculation of the first term in Eq. (93) reduces, provided eqs. (89) and (90) are satisfied, to calculate the energy gain of $\hat{\mathcal{H}}_{R}^{0}$ due to the renormalized tunnelling term

$$
\hat{V}_{R}=\sum_{\alpha k \sigma} \frac{R V_{k}}{\sqrt{\Omega}} d_{\sigma}^{\dagger} c_{\alpha k \sigma}+H . c . .
$$

The variational Hamiltonian whose ground state is the uncorrelated wavefunction $\left|\Psi_{0}\right\rangle$ has rigorously no physical meaning but for the ground state properties. However, it is common 27 to interpret it as the Hamiltonian of the quasi-particles and

$$
R^{2}=z
$$




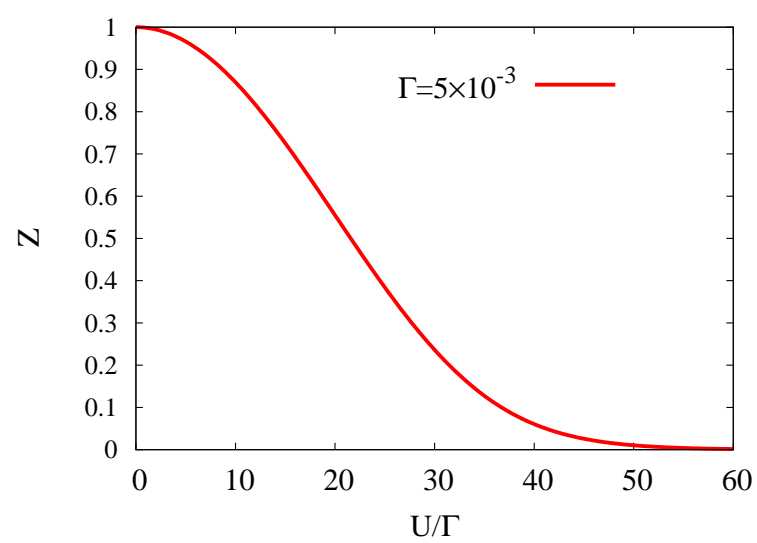

FIG. 2: (Color online) $z$, as a function of $U / \Gamma$.

as the quasi-particle weight of a single-particle excitation. Within such an assumption, the Gutzwiller approximation technique can be regarded as a tool to extract quasiparticle properties.

From now on the unit of energy is given by the conduction electron half-bandwidth $W$. The explicit value of the variational energy (referred to the ground state energy of the unperturbed system), is then given by

$$
\delta E[\Psi]=\frac{2}{\pi} z \Gamma \log \left(\frac{z \Gamma}{e}\right)+\frac{U}{4}(1-\sqrt{1-z}) .
$$

In Fig. 2 we show the value of the optimal $z$, as a function of $U$. At $U=0$ we find that $z=1$ (as expected) and has a finite curvature. When $U \rightarrow \infty$ we find that

$$
z(U) \sim \frac{1}{\Gamma} \exp \left(-\frac{\pi}{16} \frac{U}{\Gamma}\right) .
$$

Notice that at large $U$ the value of $z$ vanishes exponentially but remains finite because

$$
\left\langle\Psi_{0}\left|\hat{\mathcal{H}}_{R}^{0}\right| \Psi_{0}\right\rangle=-\frac{2}{\pi} z \Gamma \log \left(\frac{e}{z \Gamma}\right),
$$

which vanishes at $z=0$ with an infinite derivative due to the presence of $z$ in the logarithm.

We conclude this section by underlining a limit of the Gutzwiller method from a quantitative point of view. Using Eq. (99) we can define the "Gutzwiller approximation" for the Kondo temperature as

$$
T_{K}^{G} \sim z(U) \stackrel{U \gg \Gamma}{\sim} \frac{1}{\Gamma} \exp \left(-\frac{\pi}{16} \frac{U}{\Gamma}\right)
$$

Notice that $T_{K}^{G}$ differs with respect to the correct value of the Kondo temperature

$$
T_{K} \sim \exp \left(-\frac{\pi}{8} \frac{U}{\Gamma}\right)
$$

because:
- the universal prefactor in the exponent should be $\pi / 8$ and not $\pi / 16$,

- the factor $W / \Gamma$ (which is equal to $1 / \Gamma$ in our units) in Eq. (101) diverges in the infinite bandwidth limit.

The divergence of the right member of Eq. (101) for $W / \Gamma \rightarrow \infty$ reflects the unreliability of the method in this limit. In fact, when $W / \Gamma \rightarrow \infty$, the Gutzwiller approximation predicts that $z \rightarrow 1$ even if $U>\Gamma$, as can be verified directly from Eq. (98).

\section{B. Variance optimization}

For the equilibrium ground state of a system it is possible to define an infinite number of functionals with the same minimum. For example, the functionals

$$
F_{\mu}[\Psi]=E[\Psi]+\mu \sigma_{\mathcal{H}}[\Psi]
$$

being $\sigma_{\mathcal{H}}$ the variance

$$
\sigma_{\mathcal{H}}[\psi]=\left[\left\langle\psi\left|\mathcal{H}^{2}\right| \psi\right\rangle-\langle\psi|\mathcal{H}| \psi\rangle^{2}\right]^{\frac{1}{2}},
$$

are equivalent $\forall \mu>0$. Nevertheless the result obtained when the functional $F_{\mu}$ is minimized on a particular variational space depends on the factor $\mu$, and the choice has to be motivated on the basis of the specific problem considered.

In particular, if one think that his variational function is a good approximation of the ground state (and not of the excited states) of the system, instead of minimizing the energy $E$, it is sometime convenient to minimize the variance $\sigma_{\mathcal{H}}$. From the equilibrium variational principle, the smaller the energy is the better the variational state will be, but, without an exact solution, it is hard to judge how accurate the variational approximation is. On the contrary, the variance is very useful, because the smallest possible variance, equal to zero, is known a priori, and in this case the variational state represent an exact eigenstate of the Hamiltonian.

We observe that in the Gutzwiller energy-minimization procedure discussed in the previous section the optimization scheme leads to a correspondence between the $\lambda$ parameters (defining $\mathcal{P}_{d}$ through Eq. (88)) and the uncorrelated wavefunction $\left|\Psi_{0}\right\rangle$ : once the $\lambda$-parameters were defined the corresponding $\left|\Psi_{0}\right\rangle$ was the ground state of a renormalized "variational Hamiltonian" $\mathcal{H}_{R}^{0}$, with $R$ given by Eq. (95). In the appendix we show the calculation of the variance assuming such correspondence.

If, for simplicity, we assume that

$$
\begin{aligned}
& V_{k}=V_{k^{\prime}} \quad \forall k, k^{\prime} \\
& \Gamma \ll W=1,
\end{aligned}
$$

the variance is given by.

$$
\sigma_{\mathcal{H}}^{2}[\Psi]=(1-z)\left(\frac{\Gamma}{2}+\frac{12}{\pi^{2}} \Gamma^{2} z \log ^{2}\left(z^{2} \Gamma^{2}\right)\right)
$$




$$
+\frac{U}{\pi} z \sqrt{1-z} \Gamma \log \left(z^{2} \Gamma^{2}\right)+\frac{z U^{2}}{16} .
$$

Unfortunately, the minimization of the variance functional (106) respect to the allowed values of $z$ does not lead to a physically reasonable result (not shown). Our conclusion is that the minimization of the variance requires, to be effective, a more realistic trial state respect to the simple Gutzwiller-type wavefunction (87) considered here.

Following the derivation of Eq. (106) in the appendix it is clear that only for Gutzwiller's wavefunction such that the correspondence (95) between $\left|\Psi_{0}\right\rangle$ and $\mathcal{P}_{d}$ is verified the variance is finite. We underline that at half-filling such correspondence eliminates the diverging terms (for our system) even out of equilibrium, i.e. when $\left|\Psi_{0}\right\rangle$ is the ground state of

$$
\begin{aligned}
\hat{\mathcal{H}}_{R}^{0}(\Phi) & =\sum_{\alpha k \sigma} \epsilon_{k} \psi_{\alpha k \sigma}^{\dagger}(R) \psi_{\alpha k \sigma}(R)+ \\
& +\Phi \sum_{\alpha k \sigma} \frac{\alpha}{2} \psi_{\alpha k \sigma}^{\dagger}(R) \psi_{\alpha k \sigma}(R)
\end{aligned}
$$

instead of the ground state of

$$
\begin{aligned}
\hat{\mathcal{H}}_{R}^{0} & =\sum_{\alpha k \sigma} \epsilon_{k} c_{\alpha k \sigma}^{\dagger} c_{\alpha k \sigma}+\sum_{\alpha k \sigma} \frac{R V_{k}}{\sqrt{\Omega}} d_{\sigma}^{\dagger} c_{\alpha k \sigma}+H . c . \\
& \equiv \sum_{\alpha k \sigma} \epsilon_{k} \psi_{\alpha k \sigma}^{\dagger}(R) \psi_{\alpha k \sigma}(R)
\end{aligned}
$$

being

$$
\begin{aligned}
\psi_{\alpha k \sigma}^{\dagger}(R) & =c_{\alpha k \sigma}^{\dagger}+\frac{R V_{k}}{\sqrt{\Omega}} g_{d}^{R}\left(\epsilon_{k}\right) d_{\sigma}^{\dagger} \\
& +\sum_{\alpha^{\prime} k^{\prime} \sigma^{\prime}} \frac{R^{2} V_{k} V_{k^{\prime}}}{\Omega} \frac{g_{d}^{R}\left(\epsilon_{k}\right)}{\epsilon_{k}-\epsilon_{k^{\prime}}+i 0^{+}} c_{\alpha^{\prime} k^{\prime} \sigma}^{\dagger}(109
\end{aligned}
$$

the appropriate renormalized scattering operators identified by $R$.

This observation suggests that at half-filling the simple form of the trial function (87) is a reasonable variational representation not only for the ground state of our system, but also for its nonequilibrium Hershfield steady states. This observation relates with the Fermi-liquid assumption formulated in SecVI.

\section{THE GUTZWILLER APPROXIMATION OUT OF EQUILIBRIUM}

We study now the half-filled Anderson model

$$
\begin{aligned}
\hat{\mathcal{H}} & =\sum_{\alpha k \sigma} \epsilon_{k} c_{\alpha k \sigma}^{\dagger} c_{\alpha k \sigma}+\sum_{\alpha k \sigma} \frac{V_{k}}{\sqrt{\Omega}} d_{\sigma}^{\dagger} c_{\alpha k \sigma}+H . c . \\
& +\frac{U}{2}\left(\hat{n}_{d}-1\right)^{2}
\end{aligned}
$$

when it is driven out of equilibrium preparing the leads at two different chemical potentials (Fig. 11). Turning on the tunnelling interaction we know that a current starts to flow and the system, after a transient time, reaches the steady state formally defined by Eq. (10). At zero temperature the steady state is therefore the ground state of

$$
\hat{\mathcal{H}}(\Phi)=\hat{\mathcal{H}}+\Phi \hat{Y}
$$

We want to approximate the Hershfield steady state with the usual equilibrium Gutzwiller variational wavefunction

$$
|\Psi\rangle=\mathcal{P}_{d}\left|\Psi_{0}\right\rangle
$$

which satisfies conditions (89) and (90).

The average on $|\Psi\rangle$ of the non-interacting part of $\hat{\mathcal{H}}$

$$
\hat{\mathcal{H}}_{0}=\sum_{\alpha k \sigma} \epsilon_{k} c_{\alpha k \sigma}^{\dagger} c_{\alpha k \sigma}+\sum_{\alpha k \sigma} \frac{V_{k}}{\sqrt{\Omega}} d_{\sigma}^{\dagger} c_{\alpha k \sigma}+H . c .
$$

is equal to the average on $\left|\Psi_{0}\right\rangle$ of the renormalized noninteracting Hamiltonian

$$
\hat{\mathcal{H}}_{R}^{0}=\sum_{\alpha k \sigma} \epsilon_{k} c_{\alpha k \sigma}^{\dagger} c_{\alpha k \sigma}+\sum_{\alpha k \sigma} \frac{R V_{k}}{\sqrt{\Omega}} d_{\sigma}^{\dagger} c_{\alpha k \sigma}+H . c . .
$$

The difference between the equilibrium and the nonequilibrium case is that in the presence of a bias we can consider only Slater determinants belonging to $\mathcal{I}(\Phi)$. In order to guarantee the (approximated) equivalence of Eq. (80) and Eq. (72) the only reasonable state is the Hershfield steady state of the renormalized uncorrelated system (114), namely the ground state of the Hamiltonian

$$
\hat{\mathcal{H}}_{R}^{0}(\Phi)=\hat{\mathcal{H}}_{R}^{0}+\Phi \hat{Y}_{R}^{0}
$$

defined in Eq. (107). In fact, just like at equilibrium, we expect that the Hamiltonian (107) should describe weakly interacting quasiparticles in the presence of a bias. Our particular choice for the variational Slater determinant $\left|\Psi_{0}\right\rangle$ in Eq. (112) simply means that the number of left and right quasiparticle is equal to the number of left and right particles in the unperturbed system.

Summarizing, our variational choice corresponds to approximate the asymptotic time evolution operator $\Omega_{+}$ with

$$
\Omega_{+}^{G}=\mathcal{P}_{d} \Omega_{+R}^{G}
$$

being $\Omega_{+R}^{G}$ the unitary operator (to be determined variationally) such that

$$
\Omega_{+R}^{G} c_{\alpha k \sigma}^{\dagger} \Omega_{+R}^{G \dagger}=\psi_{\alpha k \sigma}^{\dagger}(R) \quad \forall \alpha, k, \sigma .
$$

The corresponding procedure amounts to minimize the following energy functional

$$
\begin{aligned}
& E_{\Phi}[\Psi]=\left\langle\Psi_{0}(\Phi)\left|\hat{\mathcal{H}}_{R}^{0}\right| \Psi_{0}(\Phi)\right\rangle \\
& +\frac{U}{2}\left\langle\Psi_{0}(\Phi)\left|\mathcal{P}_{d}^{\dagger}\left(\hat{n}_{d}-1\right)^{2} \mathcal{P}_{d}\right| \Psi_{0}(\Phi)\right\rangle,
\end{aligned}
$$




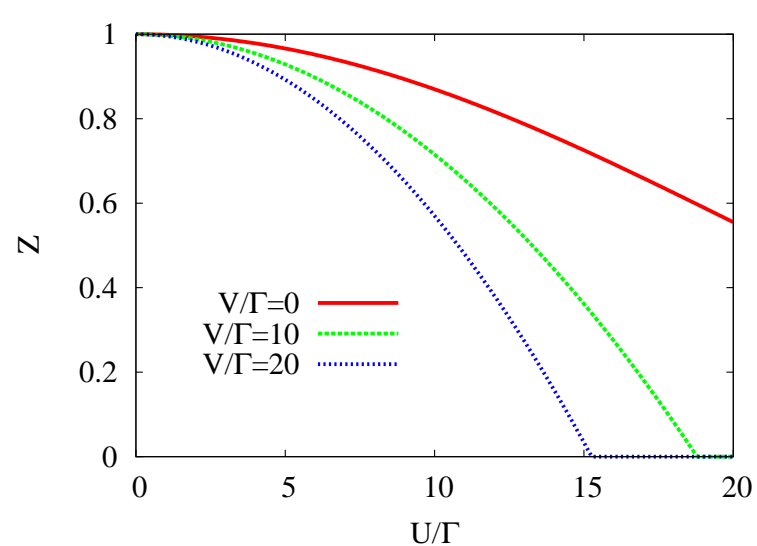

FIG. 3: (Color online) $z$ as a function of $U / \Gamma$ for $\Gamma=5 \times 10^{-3}$ and three different values of the bias $V / \Gamma$.

where $\left|\Psi_{0}(\Phi)\right\rangle$ is the ground state of $\hat{\mathcal{H}}_{R}^{0}(\Phi)$ (that satisfy the conditions (89][90)). In other words, the only difference respect to the equilibrium calculation is that we substitute the equilibrium energy gain due to the tunnelling term (96) for the energy gain due to the tunnelling term in the non equilibrium quasi-particle Hamiltonian (107). It can be easily proven that the value of the variational energy (referred to the nonequilibrium energy of the unperturbed system), is given by

$$
\delta E_{\Phi}[\Psi]=\delta \mathcal{E}_{\Phi}[\Psi]+\frac{U}{4}(1-\sqrt{1-z})
$$

where

$$
\begin{aligned}
\delta \mathcal{E}_{\Phi}[\Psi]= & -\frac{2}{\pi} \arctan (z \Gamma)+\frac{\Phi}{\pi} \arctan \left(\frac{z \Gamma}{\Phi / 2}\right) \\
& +\frac{z \Gamma}{\pi} \log \left(\frac{z^{2} \Gamma^{2}+(\Phi / 2)^{2}}{1+z^{2} \Gamma^{2}}\right)
\end{aligned}
$$

We stress that our functional, and then the value of $R$ after the optimization, depends on the bias $\Phi$. This is crucial in order to properly take into account the strong correlation effects induced by the Hubbard repulsion, i.e. to obtain the expected destruction of the Kondo resonance at finite bias (see Fig. 3).

The expression for the average of the current after the optimization is

$$
\begin{aligned}
I & =-i \sum_{k \sigma} \frac{V_{k}}{\sqrt{\Omega}}\left(\left\langle\Psi_{0}(\Phi)\left|d_{\sigma}^{\dagger} c_{k \sigma,-1}\right| \Psi_{0}(\Phi)\right\rangle-c . c .\right) \\
& =\int_{-\frac{\Phi}{2}}^{\frac{\Phi}{2}} d \epsilon \Gamma_{R}(\epsilon) \rho_{d}^{\Gamma_{R}}(\epsilon)
\end{aligned}
$$

where $\rho_{d}^{\Gamma_{R}}(\epsilon)$ is the spectral function of the dot, that is

$$
\rho_{d}^{\Gamma_{R}}(\epsilon)=\frac{1}{\pi} \frac{\Gamma_{R}}{\epsilon^{2}+\Gamma_{R}^{2}} \chi_{[-1,1]}(\epsilon)
$$

with

$$
\Gamma_{R}(\epsilon)=R^{2} \Gamma(\epsilon)
$$

having assumed that the density of states is flat and that $\Gamma \ll W=1$

$$
\begin{aligned}
\Delta(z) & =\int \frac{d \epsilon}{\pi} \frac{\Gamma(\epsilon)}{z-\epsilon} \\
\Gamma(\epsilon) & =\Gamma \chi_{[-1,1]}(\epsilon)
\end{aligned}
$$

We notice that Eq. (121) fails to describe the system accurately when $\Phi \sim U$, because it doesn't take into account the spectral contribution of the Hubbard bands. However, for the simple single-band Anderson model we can reproduce artificially the correct qualitative behaviour of the current in this regime by substituting $R^{2} \rho_{d}^{\Gamma_{R}}(\epsilon)$ with

$$
\rho_{d}^{U}(\epsilon)=R^{2} \rho_{d}^{\Gamma_{R}}(\epsilon)+\frac{1}{2}\left(1-R^{2}\right) \sum_{\alpha=-1,1} \rho_{d}^{\Gamma}\left(\epsilon-U \frac{\alpha}{2}\right)
$$

in Eq. (121).

In Fig. 4 we show the results for the conductance $G$ of the Anderson model. The obtained value of the conductance at zero bias is universal as expected, and the curvature is given by

$$
\left.\frac{d^{2} G}{d \Phi^{2}}\right|_{\Phi=0}=-\frac{1}{2 \pi\left(R^{2} \Gamma\right)^{2}} \sim-\frac{1}{\left(T_{K}^{G}\right)^{2}}
$$

- $T_{K}^{G}$ being the Kondo temperature with the incorrect prefactor predicted by the Gutzwiller method

$$
T_{K}^{G} \sim e^{-\frac{\pi}{16} \frac{U}{\Gamma}} .
$$

Nevertheless for large enough value of $U$ we found (not shown) that the conductance may become negative, which is unrealistic. In order to establish the regime of validity of our method, we note that the Fermi-liquid description that we assume is applicable only for values of the bias much lower then the Kondo temperature $T_{K}$. For the single-orbital Anderson impurity model we can calculate analytically the minimum value of the energy functional (118) when

$$
W \gg \Phi \gg \Gamma
$$

namely when Eq. (37) can be applied, so that

$$
\left\langle\Psi_{0}(\Phi)\left|\hat{\mathcal{H}}_{R}^{0}+\Phi Y_{R}^{0}\right| \Psi_{0}(\Phi)\right\rangle=\frac{2}{\pi} R^{2} \Gamma \log \left(\frac{\Phi}{2}\right) .
$$

In particular, it can be easily proven that the value of $z$ vanishes at

$$
\frac{\Phi^{*}}{2}=e^{-\frac{\pi}{16} \frac{U}{\Gamma}} \sim T_{K}^{G}
$$

that is out of the expected regime of validity of the calculation. 


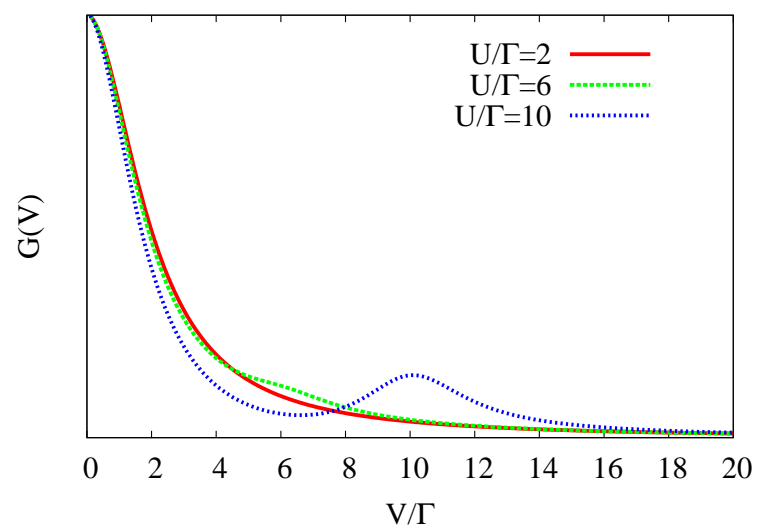

FIG. 4: (Color online) Conductance as a function of the bias $V / \Gamma$ for $\Gamma=5 \times 10^{-3}$ and three different values of $U / \Gamma$.

\section{A. Why is half-filling special?}

Let us consider the Anderson model away from particle-hole symmetry

$$
\begin{aligned}
\hat{\mathcal{H}} & =\sum_{\alpha k \sigma} \epsilon_{k} c_{\alpha k \sigma}^{\dagger} c_{\alpha k \sigma}+\sum_{\alpha k \sigma} \frac{V_{k}}{\sqrt{\Omega}} d_{\sigma}^{\dagger} c_{\alpha k \sigma}+H . c . \\
& +\epsilon_{d} \sum_{\sigma} d_{\sigma}^{\dagger} d_{\sigma}+\frac{U}{2}\left(n_{d}-1\right)^{2}
\end{aligned}
$$

by requiring $\epsilon_{d} \neq 0$. The state $\left|\psi_{0}\right\rangle$ which minimize the energy $\hat{\mathcal{H}}_{R}^{0}$ and satisfies Eq. (90)

$$
\left\langle\Psi_{0}\left|d_{\sigma}^{\dagger} d_{\sigma}\right| \Psi_{0}\right\rangle=n
$$

can be calculated within the Lagrange multipliers method, namely $\left|\psi_{0}\right\rangle$ is the ground state of the Hamiltonian

$$
\hat{\mathcal{H}}_{R}^{\mu}=\hat{\mathcal{H}}_{R}^{0}+\mu \sum_{\sigma}\left(d_{\sigma}^{\dagger} d_{\sigma}-n\right)
$$

with a proper chemical potential $\mu$.

In particular, when $\epsilon_{d}=0$ the ground state of $\hat{\mathcal{H}}_{R}^{0}$ satisfies the constraint (90) automatically, namely $\hat{\mathcal{H}}_{R}^{\mu}=$ $\hat{\mathcal{H}}_{R}^{0}$, and the corresponding non-equilibrium Hamiltonian $\hat{\mathcal{H}}_{R}^{0}(\Phi)$ automatically satisfies the constraint (90) too,

$$
\left\langle\Psi_{0}\left|d_{\sigma}^{\dagger} d_{\sigma}\right| \Psi_{0}\right\rangle=\left\langle\Psi_{0}(\Phi)\left|d_{\sigma}^{\dagger} d_{\sigma}\right| \Psi_{0}(\Phi)\right\rangle=\frac{1}{2}
$$

Let us now consider the general case $\epsilon_{d} \neq 0$. In this case

$$
\hat{\mathcal{H}}_{R}^{\mu}=\sum_{\alpha k \sigma} \epsilon_{k} \psi_{\alpha k \sigma}^{\dagger}(R) \psi_{\alpha k \sigma}(R),
$$

where $\psi_{\alpha k \sigma}^{\dagger}(R)$ where $\psi_{\alpha k \sigma}^{\dagger}(R)$ are the scattering waves constructed with renormalized hybridization $R V_{k}$, which depend on the retarded impurity Green's function

$$
g_{d R}(\epsilon)=\frac{1}{\epsilon-\mu+i R^{2} \Gamma} .
$$

We observe that, if $\mu$ is taken to be the value that satisfies the constraint (90) at equilibrium, the ground state $\left|\Psi_{0}(\Phi)\right\rangle$ of the non-equilibrium Hamiltonian

$$
\begin{aligned}
\hat{\mathcal{H}}_{R}^{0}(\Phi) & =\sum_{\alpha k \sigma} \epsilon_{k} \psi_{\alpha k \sigma}^{\dagger}(R) \psi_{\alpha k \sigma}(R) \\
& +\Phi \sum_{\alpha k \sigma} \frac{\alpha}{2} \psi_{\alpha k \sigma}^{\dagger}(R) \psi_{\alpha k \sigma}(R)
\end{aligned}
$$

is not such that

$$
\left\langle\Psi_{0}(\Phi)\left|d_{\sigma}^{\dagger} d_{\sigma}\right| \Psi_{0}(\Phi)\right\rangle=n
$$

namely it doesn't satisfy anymore (90). The procedure described for $\epsilon_{d}=0$ should then be modified without fulfilling this condition. This forces us to renounce to the mechanism that eliminates the diverging terms of the variance mentioned in Sec.VIIB, and that we interpreted as measure of merit of the variational ansatz defined in Eq. (116).

\section{CONCLUSIONS}

We have defined a variational principle based on the minimization of a bias-dependent functional of the variance for studying the steady-state zero-temperature properties of a general quantum-dot driven out of equilibrium through the application of a bias. We have proposed a similar (although approximated) method based on a "constrained" minimization of the energy.

The ideas proposed in this paper are mainly inspired by the Hershfield's point of view $\underline{19}$ that the out-ofequilibrium steady state can be regarded as the equilibrium one with an Hamiltonian $\hat{\mathcal{H}}(\Phi)$ that includes an effective nonequilibrium term proportional to the bias $\Phi \hat{Y}$. Our main result is that, equivalently, the steady state can be identified by the following conditions:

- the initial state $\rho_{0}(\Phi)$ (identified by $\Phi \hat{Y}_{0}$ ) defines a phase $\mathcal{I}(\Phi)$ of "macroscopically equivalent" states that contains also the steady state $\rho(\Phi)$

- $\rho(\Phi)$ is the "only" stationary state of the correlated Hamiltonian $\mathcal{H}$ in $\mathcal{I}(\Phi)$.

Such characterization of the nonequilibrium steady state does not require the explicit knowledge of the Hershfield's operator $\hat{Y}$, and, for this reason, we believe that it constitutes an interesting and useful formulation of the problem. It opens, in fact, a new possibility to treat nonequilibrium using equilibrium methods.

In order to test our methods, we have considered the simple single orbital Anderson impurity model at halffilling, finding a good qualitative accord with the observed behavior in quantum dots for the expected regime of validity. The choice of the variational space was, in fact, based on the the assumption that the effective Hershfield Hamiltonian $\hat{\mathcal{H}}(\Phi)$ describes a local Fermi liquid theory ${ }^{23}$ in the Noziéres sense. ${ }^{22}$ 
The ideas that we have proposed have the big advantage of being very simple, and we believe that further developments will enable us to deal with more complicated situations and variational spaces.

\section{Acknowledgments}

I am truly indebted to Prof. Michele Fabrizio for insightful discussions that allowed me to clarify several important points related to this work. Further more I thank
Prof. Giovanni Morchio, Prof. Bo Hellsing and Hugo Strand for constructive discussions and comments on the manuscript.
Appendix: Derivation of Eq. (106)

The variance of the Anderson impurity model (86) respect to our Gutzwiller variational function (87) is given by

$$
\begin{aligned}
\sigma_{\mathcal{H}}^{2} & {[\Psi]=\left(\left\langle\Psi_{0}\left|\mathcal{P}_{d}^{\dagger} \hat{T}^{2} \mathcal{P}_{d}\right| \Psi_{0}\right\rangle-\left\langle\Psi_{0}\left|\mathcal{P}_{d}^{\dagger} \hat{T} \mathcal{P}_{d}\right| \Psi_{0}\right\rangle^{2}\right) } \\
& +\left(\left\langle\Psi_{0}\left|\mathcal{P}_{d}^{\dagger} \hat{T} \hat{V} \mathcal{P}_{d}\right| \Psi_{0}\right\rangle+\left\langle\Psi_{0}\left|\mathcal{P}_{d}^{\dagger} \hat{V} \hat{T} \mathcal{P}_{d}\right| \Psi_{0}\right\rangle-2\left\langle\Psi_{0}\left|\mathcal{P}_{d}^{\dagger} \hat{T} \mathcal{P}_{d}\right| \Psi_{0}\right\rangle\left\langle\Psi_{0}\left|\mathcal{P}_{d}^{\dagger} \hat{V} \mathcal{P}_{d}\right| \Psi_{0}\right\rangle\right) \\
& +\left(\left\langle\Psi_{0}\left|\mathcal{P}_{d}^{\dagger} \hat{V}^{2} \mathcal{P}_{d}\right| \Psi_{0}\right\rangle-\left\langle\Psi_{0}\left|\mathcal{P}_{d}^{\dagger} \hat{V} \mathcal{P}_{d}\right| \Psi_{0}\right\rangle^{2}\right) \\
& +\left(\left\langle\Psi_{0}\left|\mathcal{P}_{d}^{\dagger} \hat{V} \hat{U} \mathcal{P}_{d}\right| \Psi_{0}\right\rangle+\left\langle\Psi_{0}\left|\mathcal{P}_{d}^{\dagger} \hat{U} \hat{V} \mathcal{P}_{d}\right| \Psi_{0}\right\rangle-2\left\langle\Psi_{0}\left|\mathcal{P}_{d}^{\dagger} \hat{U} \mathcal{P}_{d}\right| \Psi_{0}\right\rangle\left\langle\Psi_{0}\left|\mathcal{P}_{d}^{\dagger} \hat{V} \mathcal{P}_{d}\right| \Psi_{0}\right\rangle\right) \\
& +\left(\left\langle\Psi_{0}\left|\mathcal{P}_{d}^{\dagger} \hat{T} \hat{U} \mathcal{P}_{d}\right| \Psi_{0}\right\rangle+\left\langle\Psi_{0}\left|\mathcal{P}_{d}^{\dagger} \hat{U} \hat{T} \mathcal{P}_{d}\right| \Psi_{0}\right\rangle-2\left\langle\Psi_{0}\left|\mathcal{P}_{d}^{\dagger} \hat{T} \mathcal{P}_{d}\right| \Psi_{0}\right\rangle\left\langle\Psi_{0}\left|\mathcal{P}_{d}^{\dagger} \hat{U} \mathcal{P}_{d}\right| \Psi_{0}\right\rangle\right) \\
& +\left(\left\langle\Psi_{0}\left|\mathcal{P}_{d}^{\dagger} \hat{U} \mathcal{P}_{d}\right| \Psi_{0}\right\rangle-\left\langle\Psi_{0}\left|\mathcal{P}_{d}^{\dagger} \hat{U} \mathcal{P}_{d}\right| \Psi_{0}\right\rangle^{2}\right)
\end{aligned}
$$

Our calculation can be considerably simplified by the following considerations.

- The variance of the renormalized Hamiltonian

$$
\mathcal{H}_{0}^{R}=\hat{T}+\hat{V}_{R}
$$

on the function $\left|\Psi_{0}\right\rangle$

$$
\begin{aligned}
\sigma_{\mathcal{H}_{0}^{R}}\left[\Psi_{0}\right]= & \left(\left\langle\Psi_{0}\left|\hat{T}^{2}\right| \Psi_{0}\right\rangle-\left\langle\Psi_{0}|\hat{T}| \Psi_{0}\right\rangle^{2}\right) \\
+ & R\left(\left\langle\Psi_{0}|\hat{T} \hat{V}| \Psi_{0}\right\rangle+\left\langle\Psi_{0}|\hat{V} \hat{T}| \Psi_{0}\right\rangle\right. \\
& \left.-2\left\langle\Psi_{0}|\hat{T}| \Psi_{0}\right\rangle\left\langle\Psi_{0}|\hat{V}| \Psi_{0}\right\rangle\right) \\
+ & R^{2}\left(\left\langle\Psi_{0}\left|\hat{V}^{2}\right| \Psi_{0}\right\rangle-\left\langle\Psi_{0}|\hat{V}| \Psi_{0}\right\rangle^{2}\right)
\end{aligned}
$$

is zero, because $\left|\Psi_{0}\right\rangle$ is, by definition, the ground state of $\mathcal{H}_{0}^{R}$.

- Our variational function satisfies the Gutzwiller constraint defined in Eq. (90), so that the following equations holds:

$$
\begin{aligned}
\left\langle\Psi_{0}\left|\mathcal{P}_{d}^{\dagger} \hat{T} \mathcal{P}_{d}\right| \Psi_{0}\right\rangle & =\left\langle\Psi_{0}|\hat{T}| \Psi_{0}\right\rangle \\
\left\langle\Psi_{0}\left|\mathcal{P}_{d}^{\dagger} \hat{V} \mathcal{P}_{d}\right| \Psi_{0}\right\rangle & =R\left\langle\Psi_{0}|\hat{V}| \Psi_{0}\right\rangle
\end{aligned}
$$

- A direct calculation shows that

$$
\left\langle\Psi_{0}\left|\mathcal{P}_{d}^{\dagger} \hat{T} \hat{V} \mathcal{P}_{d}\right| \Psi_{0}\right\rangle=R\left\langle\Psi_{0}|\hat{T} \hat{V}| \Psi_{0}\right\rangle
$$

$$
\begin{aligned}
& \left\langle\Psi_{0}\left|\mathcal{P}_{d}^{\dagger} \hat{V} \hat{T} \mathcal{P}_{d}\right| \Psi_{0}\right\rangle=R\left\langle\Psi_{0}|\hat{V} \hat{T}| \Psi_{0}\right\rangle \\
& \left\langle\Psi_{0}\left|\mathcal{P}_{d}^{\dagger} \hat{T} \hat{U} \mathcal{P}_{d}\right| \Psi_{0}\right\rangle+\left\langle\Psi_{0}\left|\mathcal{P}_{d}^{\dagger} \hat{U} \hat{T} \mathcal{P}_{d}\right| \Psi_{0}\right\rangle \\
& -2\left\langle\Psi_{0}\left|\mathcal{P}_{d}^{\dagger} \hat{T} \mathcal{P}_{d}\right| \Psi_{0}\right\rangle\left\langle\Psi_{0}\left|\mathcal{P}_{d}^{\dagger} \hat{U} \mathcal{P}_{d}\right| \Psi_{0}\right\rangle=0 \\
& \left\langle\Psi_{0}\left|\mathcal{P}_{d}^{\dagger} \hat{V} \hat{U} \mathcal{P}_{d}\right| \Psi_{0}\right\rangle+\left\langle\Psi_{0}\left|\mathcal{P}_{d}^{\dagger} \hat{U} \hat{V} \mathcal{P}_{d}\right| \Psi_{0}\right\rangle \\
& \quad-2\left\langle\Psi_{0}\left|\mathcal{P}_{d}^{\dagger} \hat{U} \mathcal{P}_{d}\right| \Psi_{0}\right\rangle\left\langle\Psi_{0}\left|\mathcal{P}_{d}^{\dagger} \hat{V} \mathcal{P}_{d}\right| \Psi_{0}\right\rangle \\
& =\frac{U}{2} \lambda_{1} \lambda_{0,2}\left(1-\lambda_{0,2}^{2}\right)\left\langle\Psi_{0}|\hat{V}| \Psi_{0}\right\rangle \\
& \left\langle\Psi_{0}\left|\mathcal{P}_{d}^{\dagger} \hat{U}^{2} \mathcal{P}_{d}\right| \Psi_{0}\right\rangle-\left\langle\Psi_{0}\left|\mathcal{P}_{d}^{\dagger} \hat{U} \mathcal{P}_{d}\right| \Psi_{0}\right\rangle^{2} \\
& =\left(\frac{U}{2}\right)^{2} \frac{\lambda_{0,2}^{2}}{2}\left(1-\frac{\lambda_{0,2}^{2}}{2}\right)
\end{aligned}
$$

Taking the difference between Eq. A.1 and Eq. A.3 and using the above equations we obtain that

$$
\begin{aligned}
\sigma_{\mathcal{H}}^{2}[\Psi] & =\left(\left\langle\Psi_{0}\left|\mathcal{P}_{d}^{\dagger} \mathcal{P}_{d} \hat{T}^{2}\right| \Psi_{0}\right\rangle-\left\langle\Psi_{0}\left|\hat{T}^{2}\right| \Psi_{0}\right\rangle\right) \\
& +\left(\left\langle\Psi_{0}\left|\mathcal{P}_{d}^{\dagger} \hat{V}^{2} \mathcal{P}_{d}\right| \Psi_{0}\right\rangle-R^{2}\left\langle\Psi_{0}\left|\hat{V}^{2}\right| \Psi_{0}\right\rangle\right) \\
& +\frac{U}{2} \lambda_{1} \lambda_{0,2}\left(1-\lambda_{0,2}^{2}\right)\left\langle\Psi_{0}|\hat{V}| \Psi_{0}\right\rangle \\
& +\left(\frac{U}{2}\right)^{2} \frac{\lambda_{0,2}^{2}}{2}\left(1-\frac{\lambda_{0,2}^{2}}{2}\right) .
\end{aligned}
$$


Let us consider now the first term in Eq. A.9, which is equal to the sum of all the Wick contractions in which the operators $\mathcal{P}^{\dagger} \mathcal{P}$ and $\hat{T}^{2}$ are connected by two or four "legs".

A direct calculation shows that

$$
\begin{aligned}
\mathcal{P}_{d}^{\dagger} \mathcal{P}_{d} & =\lambda_{0,2}^{2}-\left(\lambda_{0,2}^{2}-\lambda_{1}^{2}\right)\left(d_{\uparrow}^{\dagger} d_{\uparrow}+d_{\downarrow}^{\dagger} d_{\downarrow}\right) \\
& +2\left(\lambda_{0,2}^{2}-\lambda_{1}^{2}\right) d_{\uparrow}^{\dagger} d_{\uparrow} d_{\downarrow}^{\dagger} d_{\downarrow} .
\end{aligned}
$$

Using Eq. A.10 it can be easily verified that the sum of all the two-legs contraction between $\mathcal{P}_{d}^{\dagger} \mathcal{P}_{d}$ and $\hat{T}^{2}$ is zero. The four legs contribution can be calculated using Wick's theorem. The result is

$$
\begin{gathered}
\left\langle\Psi_{0}\left|\mathcal{P}_{d}^{\dagger} \mathcal{P}_{d} \hat{T}^{2}\right| \Psi_{0}\right\rangle-\left\langle\Psi_{0}\left|\hat{T}^{2}\right| \Psi_{0}\right\rangle \\
=\left(\lambda_{0,2}^{2}-\lambda_{1}^{2}\right) \mathcal{A}^{2}\left(\mathcal{P}_{d}\right)
\end{gathered}
$$

where

$$
\mathcal{A}\left(\mathcal{P}_{d}\right) \equiv \sum_{k \alpha \sigma} \epsilon_{k}\left|\left\langle\Psi_{0}\left|d_{\sigma}^{\dagger} c_{\alpha k \sigma}\right| \Psi_{0}\right\rangle\right|^{2} .
$$

Let us consider the second term in Eq. A.9. It can be verified that

$$
\begin{aligned}
& \left\langle\Psi_{0}\left|\mathcal{P}_{d}^{\dagger} \hat{V}^{2} \mathcal{P}_{d}\right| \Psi_{0}\right\rangle-R^{2}\left\langle\Psi_{0}\left|\mathcal{P}_{d}^{\dagger} \hat{V}^{2} \mathcal{P}_{d}\right| \Psi_{0}\right\rangle \\
& =\left(1-R^{2}\right)\left(\sum_{k \alpha} \frac{V_{k}^{2}}{\Omega}+3 \frac{\mathcal{V}^{2}\left(\mathcal{P}_{d}\right)}{R^{2}}\right) ;
\end{aligned}
$$

where

$$
\mathcal{V}\left(\mathcal{P}_{d}\right) \equiv \sum_{k \alpha \sigma} \frac{R V_{k}}{\sqrt{\Omega}}\left\langle\Psi_{0}\left|c_{\alpha k \sigma}^{\dagger} d_{\sigma}\right| \Psi_{0}\right\rangle+c . c . .
$$

The above calculations lead to the following expression for the variance:

$$
\sigma_{\mathcal{H}}^{2}[\Psi]=\left(\lambda_{0,2}^{2}-\lambda_{1}^{2}\right) \mathcal{A}^{2}\left(\mathcal{P}_{d}\right)
$$

$$
\begin{aligned}
& +\left(1-R^{2}\right)\left(\sum_{k \alpha} \frac{V_{k}^{2}}{\Omega}+3 \frac{\mathcal{V}^{2}\left(\mathcal{P}_{d}\right)}{R^{2}}\right) \\
& +\frac{U}{2}\left(1-\lambda_{0,2}^{2}\right) \mathcal{V}\left(\mathcal{P}_{d}\right) \\
& +\left(\frac{U}{2}\right)^{2} \frac{\lambda_{0,2}^{2}}{2}\left(1-\frac{\lambda_{0,2}^{2}}{2}\right) \\
& \equiv \sigma_{\mathcal{H}_{0}}^{2}[\Psi]+\delta \sigma_{\text {int }}^{2}[\Psi] .
\end{aligned}
$$

Notice that the functional relation (95) between $\left|\Psi_{0}\right\rangle$ and $\mathcal{P}_{d}$ has been responsible of the cancellation of the $\hat{T} \hat{V}$ terms in Eq. A.1 , that are extensive quantities.

It can be easily proven that the minimum condition of $\sigma_{\mathcal{H}_{0}}^{2}$ in the not projected state implies that

$$
\mathcal{A}^{2}\left(\mathcal{P}_{d}\right)=0
$$

The explicit value of $\mathcal{V}\left(\mathcal{P}_{d}\right)$ can be simply obtained replacing $\Gamma$ with $z \Gamma$ (see Eq. (97)) in Eq. (34).

If, for simplicity, we assume that

$$
\begin{aligned}
& V_{k}=V_{k^{\prime}} \quad \forall k, k^{\prime} \\
& \Gamma \ll W=1,
\end{aligned}
$$

it can be easily verified that the variance is given by

$$
\begin{aligned}
\sigma_{\mathcal{H}}^{2}[\Psi] & =(1-z)\left(\frac{\Gamma}{2}+\frac{12}{\pi^{2}} \Gamma^{2} z \log ^{2}\left(z^{2} \Gamma^{2}\right)\right) \\
& +\frac{U}{\pi} z \sqrt{1-z} \Gamma \log \left(z^{2} \Gamma^{2}\right)+\frac{z U^{2}}{16},
\end{aligned}
$$

that coincides with Eq. (106).
1 D. Goldhaber-Gordon, H. Shtrikman, D. Mahalu, D. Abusch-Magder, U. Meirav, and M. A. Kastner, Nature 391, 156 (1998).

2 A. Smogunov, A. Dal Corso, A. Delin, R. Weht, and E. Tosatti, Nature 3, 22 (2008).

3 A. Smogunov, A. Dal Corso, and E. Tosatti, Physical Review B (Condensed Matter and Materials Physics) 73, 075418 (2006)

4 A. Smogunov, A. Dal Corso, and E. Tosatti, Physical Review B (Condensed Matter and Materials Physics) 78, 014423 (2008).

${ }^{5}$ K. G. Wilson, Rev. Mod. Phys. 47, 773 (1975).

${ }^{6}$ R. Bulla, T. A. Costi, and T. Pruschke, Reviews of Modern Physics 80, 395 (2008).

${ }^{7}$ H. Schoeller and J. König, Phys. Rev. Lett. 84, 3686 (2000).

8 P. Lucignano, R. Mazzarello, A. Smogunov, M. Fabrizio, and E. Tosatti, Nature Materials 8, 563 (2009).

9 D. Jacob, K. Haule, and G. Kotliar, Physical Review Letters 103, 016803 (2009).

${ }^{10}$ H. Schoeller and J. König, Phys. Rev. Lett. 84, 3686 (2000).

11 J. E. Han and R. J. Heary, Physical Review Letters 99, 236808 (2007).

12 S. Weiss, J. Eckel, M. Thorwart, and R. Egger, Physical Review B (Condensed Matter and Materials Physics) 77, 195316 (2008).

${ }^{13}$ L. Mühlbacher and E. Rabani, Physical Review Letters 100, 176403 (2008).

14 P. Werner, T. Oka, and A. J. Millis, Physical Review B (Condensed Matter and Materials Physics) 79, 035320 (2009).

15 M. Schirò and M. Fabrizio, Physical Review B (Condensed Matter and Materials Physics) 79, 153302 (2009). 
16 F. B. Anders, Physical Review Letters 101, 066804 (2008).

17 M. C. Gutzwiller, Phys. Rev. 134, A923 (1964).

18 M. C. Gutzwiller, Phys. Rev. 137, A1726 (1965).

19 S. Hershfield, Phys. Rev. Lett. 70, 2134 (1993).

20 J. E. Han, Physical Review B 75, 125122 (2007).

${ }^{21}$ G. L. Sewell, Quantum Theory of Collective Phenomena (Clarendon Press, 1986).

22 Nozières, Journal of Low Temperature Physics 17 (1974)

23 M. Pustilnik and L. Glazman, Journal of Physics: Condensed Matter 16, R513 (2004).
24 A. C. Hewson, The Kondo Problem to Heavy Fermions (Cambridge University Press, 1997).

25 Fazekas and Müller-Hartmann, Z. Phys. B 85, 285 (1991).

26 P. Fazekas and B. H.Brandow, Phys. Scr. 36, 809 (1987).

27 J. Bünemann, F. Gebhard, and R. Thul, Phys. Rev. B 67, 075103 (2003).

28 The physical meaning of (12) is that the steady state can be reached only when all terms of $\hat{Y}_{0}$ that do not commute with the Hamiltonian $\hat{\mathcal{H}}$ have been filtered out. 